\title{
On the Dirichlet divisor problem in short intervals
}

\author{
Aleksandar Ivić and Wenguang Zhai *
}

\begin{abstract}
We present several new results involving $\Delta(x+U)-\Delta(x)$, where $U=o(x)$ and$$
\Delta(x):=\sum_{n \leqslant x} d(n)-x \log x-(2 \gamma-1) x
$$

is the error term in the classical Dirichlet divisor problem.

\section{Introduction}

Define as usual

$$
\Delta(x):=\sum_{n \leqslant x} d(n)-x \log x-(2 \gamma-1) x,
$$

where $d(n)=\sum_{\delta \mid n} 1$ is the sum of all positive divisors of $n$, and $\gamma=$ $-\Gamma^{\prime}(1)=0.5772157 \ldots$ is Euler's constant. Dirichlet first proved in the 19 th century that $\Delta(x)=O\left(x^{1 / 2}\right)$. The exponent $1 / 2$ was subsequently improved by many authors. The latest result reads

$$
\Delta(x) \ll x^{131 / 416}(\log x)^{26947 / 8320}, \frac{131}{416}=0.314903 \ldots,
$$

which was obtained by Huxley [6]. For $\Delta(x)$ we have the following wellknown conjecture.

Conjecture 1. For any $\varepsilon>0$

$$
\Delta(x)=O_{\varepsilon}\left(x^{1 / 4+\varepsilon}\right) .
$$

Conjecture 1 is supported by the classical mean-square result

$$
\int_{1}^{T} \Delta^{2}(x) \mathrm{d} x=\frac{(\zeta(3 / 2))^{4}}{6 \pi^{2} \zeta(3)} T^{3 / 2}+F(T)
$$

${ }^{*}$ Wenguang Zhai is supported by National Natural Science Foundation of China (Grant No. 11171344) and Natural Science Foundation of Beijing (No. 1112010) 
with $F(T)=O_{\varepsilon}\left(T^{5 / 4+\varepsilon}\right)$, proved by Cramér [1]. His result incidentally also shows that $\Delta(x)=o\left(x^{1 / 4}\right)$ cannot hold as $x \rightarrow \infty$. Here and later $\varepsilon(>0)$ denotes constants which may be arbitrarily small, but are not necessarily the same ones at each occurrence, while $O_{a, b, \ldots}$ means that the implied $O$-constant depends on $a, b, \ldots$ The estimate $F(T)=O_{\varepsilon}\left(T^{5 / 4+\varepsilon}\right)$ was improved to $O\left(T \log ^{5} T\right)$ by Tong [19], to $O\left(T \log ^{4} T\right)$ by Preissmann [17], and recently to $O\left(T \log ^{3} T \log \log T\right)$ by Lau and Tsang [15].

Conjecture 1 is also supported by the upper bound estimate (see Ivić [7] and [8])

$$
\int_{1}^{T}|\Delta(x)|^{A} \mathrm{~d} x \ll_{\varepsilon} T^{1+A / 4+\varepsilon},
$$

where $0 \leqslant A \leqslant 35 / 4$. The exponent $35 / 4$ can be replaced by $262 / 27$ if we substitute Huxley's exponent 131/416 into Ivić's machinery. For this kind of estimate, we have the following conjecture.

Conjecture 2. The estimate (1.4) holds for any $A>0$.

Remark 1. Obviously if Conjecture 1 is true, then Conjecture 2 is also true. It is easy to show that if Conjecture 2 is true, then Conjecture 1 is also true. Hence these two conjectures are equivalent.

For the asymptotic formulae of higher power moments of $\Delta(x)$ see, for example, the papers of Ivić-Sargos [12], Tsang [20] and Zhai [24].

\section{Sign changes of $\Delta(x)$ and a result of Jutila}

Suppose $T$ is a large parameter. Ivić 9 proved that there exists a positive constant $C>0$ such that $\Delta(x)$ changes its sign on $[T, T+C \sqrt{T}]$. More precisely, one can find $x_{1}, x_{2} \in[T, T+C \sqrt{T}]$ such that $\Delta\left(x_{1}\right)>c_{1} T^{1 / 4}$ and $\Delta\left(x_{2}\right)<-c_{2} T^{1 / 4}$ hold respectively. This fact was proved independently in Heath-Brown and Tsang [5].

Heath-Brown and Tsang [5] also proved that the above result is almost best possible. Actually they proved the following theorem.

Theorem A. In the interval $[T, 2 T]$ there are $\gg T^{1 / 2} \log ^{5} T$ subintervals of length $\gg T^{1 / 2} \log ^{-5} T$ such that on each subinterval one has $|\Delta(x)| \geqslant$ $c_{3} T^{1 / 4}$ for some $c_{3}>0$.

In order to prove Theorem A, Heath-Brown and Tsang used a classical result of Jutila 14 on the divisor problem in short intervals. This is 
Theorem B. If $T \geqslant 2$ and $1 \leqslant U \ll T^{1 / 2} \ll H \leqslant T$, then

$$
\begin{aligned}
\int_{T}^{T+H} & (\Delta(x+U)-\Delta(x))^{2} \mathrm{~d} x \\
= & \frac{1}{4 \pi^{2}} \sum_{n \leqslant \frac{T}{2 U}} \frac{d^{2}(n)}{n^{3 / 2}} \int_{T}^{T+H} x^{1 / 2}\left|\exp \left(2 \pi i(n / x)^{1 / 2} U\right)-1\right|^{2} \mathrm{~d} x \\
+ & O_{\varepsilon}\left(T^{1+\varepsilon}+H U^{1 / 2} T^{\varepsilon}\right) .
\end{aligned}
$$

Note that the terminology "divisor problem in short intervals" refers to the fact that in (2.1) we have $\Delta(x+U)-\Delta(x)$ with $U=o(T)$ as $T \rightarrow \infty$, hence the interval $[x, x+U]$ is "short". In the case when $H=T$, the first author [11] sharpened (2.1) to an explicit asymptotic formula. The term $T^{1+\varepsilon}$ in (2.1) can be replaced by $T \log ^{4} T$ if we use the method of Preissmann [17] in conjunction with the proof of Jutila [14].

We have the well-known asymptotic formula (see e.g., [8, Chapter 4])

$$
\sum_{n \leqslant x} d^{2}(n)=x P(\log x)+O_{\varepsilon}\left(x^{1 / 2+\varepsilon}\right)
$$

where $P(t)$ is a suitable polynomial of degree three in $t$. Hence from (2.2) and (2.1) with $T^{1+\varepsilon}$ replaced by $T \log ^{4} T$ one gets, for $1 \leqslant U \leqslant T^{1 / 2} / 2 \ll H \leqslant T$, that

$$
\int_{T}^{T+H}(\Delta(x+U)-\Delta(x))^{2} \mathrm{~d} x \ll H U \log ^{3} \frac{\sqrt{T}}{U}+T \log ^{4} T .
$$

With the help of (2.3), Heath-Brown and Tsang proved the following Lemma 2.1, which combined with the results on the moments of $\Delta(x)$ gives Theorem A.

Lemma 2.1. Suppose that $2 \leqslant U \leqslant T^{1 / 2}$. Then

$$
\int_{T}^{2 T} \max _{0 \leqslant u \leqslant U}|\Delta(x+u)-\Delta(x)|^{2} \mathrm{~d} x \ll T U \log ^{5} T .
$$

Remark 2. Heath-Brown and Tsang did not prove Lemma 2.1 for $\Delta(x)$ directly. Actually they proved Lemma 2.1 with $\Delta(x)$ replaced by

$$
\left.E(T):=\int_{0}^{T}\left|\zeta\left(\frac{1}{2}+i t\right)\right|^{2} \mathrm{~d} t-T(\log (T /(2 \pi))+2 \gamma-1)\right),
$$

which represents the error term in the asymptotic formula of $\zeta(s)$ on the "critical line" $\Re s=1 / 2$. However the proof for $\Delta(x)$ is very similar, even a little simpler.

The formula (2.1) led Jutila [14] to propose 
Conjecture 3. For any $0<\varepsilon<1 / 4$ and $x^{\varepsilon} \leqslant U \leqslant x^{1 / 2-\varepsilon}$ we have

$$
\Delta(x+U)-\Delta(x) \ll_{\varepsilon} x^{\varepsilon} \sqrt{U}
$$

This conjecture is much stronger than the unconditional estimate

$$
\Delta(x+U)-\Delta(x) \ll_{\varepsilon} x^{\varepsilon} U \quad(1 \ll U \leqslant x),
$$

which easily follows from the definition of $\Delta(x)$ and the elementary bound $d(n) \ll_{\varepsilon} n^{\varepsilon}$. It is curious that (2.6) has not been proved yet by the use of Voronoï's explicit formula for $\Delta(x)$ (see e.g., [8, Chapter 3]). On the other hand, from (1.1) one obtains by trivial estimation

$$
\Delta(x+U)-\Delta(x) \ll_{\varepsilon} x^{131 / 416+\varepsilon} \quad(1 \ll U \ll x) .
$$

\section{Bounds for $\Delta(x+U)-\Delta(x)$}

In this section we shall present new results on the estimation of the difference $\Delta(x+U)-\Delta(x)$, both pointwise and in the statistical sense, by giving an upper bound for the occurrence of large values. Our pointwise bounds are obtained without the use of the sophisticated exponential sum techniques which lead to (1.1).

Theorem 1. We have

$$
\Delta(x+U)-\Delta(x) \ll_{\varepsilon} x^{1 / 4+\varepsilon} U^{1 / 4} \quad\left(1 \ll U \ll x^{3 / 5}\right),
$$

and

$$
\Delta(x+U)-\Delta(x) \ll_{\varepsilon} x^{2 / 9+\varepsilon} U^{1 / 3} \quad\left(1 \ll U \ll x^{2 / 3}\right) .
$$

Moreover, suppose that

$$
\left|\Delta\left(x_{r}+U\right)-\Delta\left(x_{r}\right)\right| \geqslant V \gg U^{1 / 2}(\gg 1) \quad(r=1, \ldots, R-1),
$$

where $X / 2 \leqslant x_{1}<\ldots<x_{R} \leqslant X,\left|x_{r}-x_{s}\right| \geqslant V$ for $r \neq s$. If $(\kappa, \lambda)$ is an exponent pair for which $\kappa \neq 0$, then for $X^{\lambda-\kappa} \leqslant V^{3+2 \lambda-2 \kappa} U^{-2}$ we have

$$
R \ll_{\varepsilon} X^{\varepsilon}\left(X V^{-5} U^{2}+X^{(\kappa+\lambda) / \kappa} U^{(2 \kappa+2) / \kappa} V^{-(3+4 \kappa+2 \lambda) / \kappa}\right) .
$$

Corollary 3.1. If we take in (3.4) the exponent pairs

$$
(\kappa, \lambda)=(1 / 2,1 / 2),(2 / 7,4 / 7),(1 / 6,4 / 6)
$$


we obtain

$$
\begin{array}{cc}
R \ll_{\varepsilon} X^{\varepsilon}\left(X V^{-5} U^{2}+X^{2} U^{6} V^{-12}\right) & \left(V \geqslant U^{2 / 3}\right), \\
R \ll_{\varepsilon} X^{\varepsilon}\left(X V^{-5} U^{2}+X^{3} U^{9} V^{-37 / 2}\right) & \left(V \geqslant U^{14 / 25} X^{225}\right), \\
R \ll_{\varepsilon} X^{\varepsilon}\left(X V^{-5} U^{2}+X^{5} U^{14} V^{-30}\right) & \left(V \geqslant U^{1 / 2} X^{18}\right) .
\end{array}
$$

respectively.

For the definition and properties of (one-dimensional) exponent pairs, see Ivić [8] or Graham-Kolesnik [2]. If in the above estimates one could discard the second term and retain only the term $X V^{-5} U^{2}$, this would imply

$$
\int_{1}^{X}(\Delta(x+U)-\Delta(x))^{4} \mathrm{~d} x \ll_{\varepsilon} X^{1+\varepsilon} U^{2}
$$

in a suitable range for $U$, which is a conjecture of M. Jutila [14].

Proof. For the proof of Theorem 1 we need the following well-known truncated form of the Voronoï formula for $\Delta(x)$ (see e.g., [8, Chapter 3]).

Lemma 3.1. For $1 \ll N \ll x$ we have

$$
\Delta(x)=\frac{x^{1 / 4}}{\sqrt{2} \pi} \sum_{n \leqslant N} d(n) n^{-3 / 4} \cos (4 \pi \sqrt{n x}-\pi / 4)+O_{\varepsilon}\left(x^{1 / 2+\varepsilon} N^{-1 / 2}\right) .
$$

Thus setting $f(x):=x^{1 / 4} \cos (4 \pi \sqrt{n x}-\pi / 4)$ we have from (3.5)

$$
\begin{aligned}
\Delta(x+U)-\Delta(x) & =\frac{1}{\pi \sqrt{2}} \sum_{n \leqslant N} d(n) n^{-3 / 4} \int_{x}^{x+U} f^{\prime}(v) \mathrm{d} v+O_{\varepsilon}\left(x^{1 / 2+\varepsilon} N^{-1 / 2}\right) \\
& =\frac{1}{4 \pi \sqrt{2}} \int_{x}^{x+U} v^{-3 / 4} \sum_{n \leqslant N} d(n) n^{-3 / 4} \cos (4 \pi \sqrt{n v}-\pi / 4) \mathrm{d} v \\
& -\sqrt{2} \int_{x}^{x+U} v^{-1 / 4} \sum_{n \leqslant N} d(n) n^{-1 / 4} \sin (4 \pi \sqrt{n v}-\pi / 4) \mathrm{d} v \\
& +O_{\varepsilon}\left(x^{1 / 2+\varepsilon} N^{-1 / 2}\right)=\frac{1}{4 \pi \sqrt{2}} I_{1}-\sqrt{2} I_{2}+O_{\varepsilon}\left(x^{1 / 2+\varepsilon} N^{-1 / 2}\right),
\end{aligned}
$$

say. Also note that $I_{1}$ and $I_{2}$ are similar in structure, but $I_{1}$ is of a lower order of magnitude, so it suffices to estimate $I_{2}$. By Hölder's inequality for integrals we have, for $k \in \mathbb{N}$,

$$
I_{2} \ll \max _{K} \frac{\log x}{x^{1 / 4}} U^{1-1 / k}\left(\int_{x}^{x+U}\left|\sum_{K<n \leqslant K^{\prime} \leqslant 2 K} \frac{d(n)}{n^{1 / 4}} \exp (4 \pi i \sqrt{n x})\right|^{k} \mathrm{~d} v\right)^{1 / k},
$$

where the maximum is taken over $O(\log x)$ values of $K \ll N$. We shall use (3.6) with $k=2$ and $k=4$ to obtain (3.1) and (3.2), respectively. 
When $k=2$ the integral in (3.6) equals

$$
\begin{aligned}
& \int_{x}^{x+U} \sum_{K<m, n \leqslant K^{\prime}} d(m) d(n)(m n)^{-1 / 4} \exp (4 \pi i(\sqrt{m}-\sqrt{n}) \sqrt{v}) \mathrm{d} v \\
& \ll U \sum_{K<n \leqslant 2 K} d^{2}(n) n^{-1 / 2}+\sum_{K<m \neq n \leqslant 2 K} \frac{d(m) d(n) x^{1 / 2}}{(m n)^{1 / 4}|\sqrt{m}-\sqrt{n}|} \\
& \ll_{\varepsilon} U K^{1 / 2} \log ^{3} K+K^{1+\varepsilon} x^{1 / 2},
\end{aligned}
$$

where we used trivial estimation for the terms with $m=n$, and otherwise the standard first derivative test (see e.g., Lemma 2.1 of [8]). Therefore we obtain

$$
\begin{aligned}
I_{2} & \ll_{\varepsilon} U^{1 / 2} x^{\varepsilon-1 / 4}\left(U^{1 / 2} N^{1 / 4}+N^{1 / 2} x^{1 / 4}\right) \\
& \ll_{\varepsilon} x^{\varepsilon}\left(U N^{1 / 4} x^{-1 / 4}+U^{1 / 2} N^{1 / 2}\right) .
\end{aligned}
$$

This gives

$$
\Delta(x+U)-\Delta(x) \ll_{\varepsilon} x^{\varepsilon}\left(U N^{1 / 4} x^{-1 / 4}+U^{1 / 2} N^{1 / 2}+x^{1 / 2} N^{-1 / 2}\right) .
$$

If we choose $N=(x / U)^{1 / 2}$, then it follows that

$$
\Delta(x+U)-\Delta(x) \ll_{\varepsilon} x^{\varepsilon}\left(x^{1 / 4} U^{1 / 4}+U^{7 / 8} x^{-1 / 8}\right) \ll_{\varepsilon} x^{1 / 4+\varepsilon} U^{1 / 4}
$$

for $1 \ll U \ll x^{3 / 5}$, as asserted by (3.1). Note that $x^{1 / 4} U^{1 / 4} \leqslant U$ for $U \geqslant x^{1 / 3}$, hence for $x^{1 / 3} \leqslant U \leqslant x^{3 / 5}$ we obtain an improvement over (2.6), without the use of exponential sum techniques.

When $k=4$ we use the technique of the proof of (1.12) of Ivić-Zhai [13], based on an arithmetic result of Robert-Sargos [18] involving four square roots, so we omit the details. The integral in (3.6) is

$$
\ll_{\varepsilon} x^{\varepsilon} U\left(K^{5 / 2} x^{1 / 2} U^{-1}+K\right) .
$$

Hence from (3.6) we infer that

$$
\Delta(x+U)-\Delta(x) \ll_{\varepsilon} x^{\varepsilon}\left(x^{-1 / 8} N^{5 / 8} U^{3 / 4}+x^{-1 / 4} U N^{1 / 4}+x^{1 / 2} N^{-1 / 2}\right) .
$$

The choice of $N$ this time will be $N=x^{5 / 9} U^{-2 / 3}$, valid for $1 \ll U \leqslant x^{5 / 6}$. Thus

$$
\Delta(x+U)-\Delta(x) \ll_{\varepsilon} x^{\varepsilon}\left(x^{2 / 9} U^{1 / 3}+U^{5 / 6} x^{-1 / 9}\right) \ll_{\varepsilon} x^{2 / 9+\varepsilon} U^{1 / 3}
$$

for $1 \ll U \leqslant x^{2 / 3}$, as asserted by (3.2). 
It remains to prove (3.4). We shall use the method of [7], also used in Chapter 13 of [8]. From Lemma 3.1 (taking $N=X^{1+\varepsilon} V^{-2}$ ) and the condition (3.3) we obtain, for $r=1, \ldots, R$,

$$
\begin{aligned}
1 & \ll x^{-1 / 4} V^{-1} \int_{x_{r}}^{x_{r}+U}\left|\sum_{n \leqslant X^{1+\varepsilon} V^{-2}} d(n) n^{-1 / 4} e(2 \sqrt{n v})\right| \mathrm{d} v \\
& \ll x^{-1 / 4} V^{-1} U\left|\sum_{n \leqslant X^{1+\varepsilon} V^{-2}} d(n) n^{-1 / 4} e\left(2 \sqrt{n t_{r}}\right)\right|,
\end{aligned}
$$

where $e(z)=\exp (2 \pi i z)$, and $t_{r}$ is the point from $\left[x_{r}, x_{r}+U\right]$ where the integral above attains its maximum. Hence we may consider the system of points

$$
X / 3 \leqslant t_{1}<\ldots<t_{R} \leqslant 4 X / 3, \quad\left|t_{r}-t_{s}\right| \gg V \quad(r \neq s)
$$

such that

$$
1 \ll X^{-1 / 4} V^{-1} U\left|\sum_{n \leqslant X^{1+\varepsilon} V^{-2}} d(n) n^{-1 / 4} e\left(2 \sqrt{n t_{r}}\right)\right| \quad(r=1, \ldots, R) .
$$

Summation of (3.7) over $r$ and an application of the Halász-Montgomery inequality (see e.g., the Appendix of [8]) give

$$
\begin{aligned}
R & \ll X^{-1 / 2} V^{-2} U^{2} \log X \max _{M \leqslant X^{1+\varepsilon} V^{-2}} \sum_{r \leqslant R}\left|\sum_{M<n \leqslant 2 M} d(n) n^{-1 / 4} e\left(2 \sqrt{n t_{r}}\right)\right|^{2} \\
& \ll_{\varepsilon} X^{\varepsilon-1 / 2} V^{-2} U^{2} \max _{M \leqslant X^{1+\varepsilon} V^{-2}} M^{1 / 2} \max _{s \leqslant R}\left(M+\sum_{r \leqslant R, r \neq s} \frac{M^{1 / 2} X^{1 / 2}}{\left|t_{r}-t_{s}\right|}\right. \\
& \left.+\sum_{r \leqslant R} X_{0}^{\kappa} X^{-\kappa / 2} M^{-\kappa / 2+\lambda}\right) \\
& \ll_{\varepsilon} X^{1+\varepsilon} V^{-5} U^{2}+R X_{0}^{\kappa} X^{-1 / 2-\kappa / 2+\varepsilon} V^{-2} U^{2} X^{\lambda-\kappa / 2+1 / 2} V^{-2 \lambda+\kappa-1} \\
& \ll_{\varepsilon} X^{1+\varepsilon} V^{-5} U^{2}+R X_{0}^{\kappa} X^{\lambda-\kappa+\varepsilon} U^{2} V^{-2 \lambda+\kappa-3} .
\end{aligned}
$$

This in fact holds if $\left|t_{r}-t_{s}\right| \leqslant X_{0}$, namely if we estimate the number of points $R=R_{0}$, say, in an subinterval of $[X / 3,4 X / 3]$ of length $\leqslant X_{0}$ for a given $X_{0}$ to be determined a little later. Here we used the estimate

$$
\sum_{M<n \leqslant 2 M} e(2 \sqrt{n x}) \ll x^{\kappa / 2} M^{\lambda-\kappa / 2},
$$

where $(\kappa, \lambda)$ is an exponent pair. It follows that

$$
R_{0} \ll_{\varepsilon} X^{1+\varepsilon} V^{-5} U^{2}
$$

provided that

$$
X_{0}^{\kappa} X^{\lambda-\kappa+\varepsilon} U^{2} V^{-2 \lambda+\kappa-3} \ll 1
$$


which is satisfied with the choice $(\kappa>0)$

$$
X_{0}=X^{(\kappa-\lambda) / \kappa+\varepsilon} U^{-2 / \kappa} V^{(3-\kappa+2 \lambda) / \kappa}
$$

whence

$$
\begin{aligned}
R & \ll R_{0}\left(1+X / X_{0}\right) \\
& \ll \varepsilon X^{1+\varepsilon} V^{-5} U^{2}+X^{2+\varepsilon} V^{-5} U^{2} X^{(\lambda-\kappa) / 2} U^{2 / \kappa} V^{(-3+\kappa-2 \lambda) / \kappa},
\end{aligned}
$$

which implies (3.4) of Theorem 1. Since $V \leqslant\left|t_{r}-t_{s}\right| \leqslant X_{0}(r \neq s)$, we have yet to check that $V \leqslant X_{0}$, which is true if $X^{\lambda-\kappa} \leqslant V^{3+2 \lambda-2 \kappa} U^{-2}$ and, in view of (3.9), this is the condition given in the formulation of Theorem 1.

\section{A new conjecture on $\Delta(x+u)-\Delta(x)$}

We note that Jutila's result (2.1) holds on the interval $[T, T+H]$ with $T^{1 / 2} \ll$ $H \ll T$. But Lemma 2.1 is a result on the interval $[T, 2 T]$. Comparing $(2.3)$ and (2.4) it is natural to ask if we can find a short interval type result of (2.4) similar to (2.3). Here we propose the following Conjecture 3 about this kind of estimate.

Conjecture 4. Suppose

$$
\log T \leqslant U \leqslant T^{1 / 2} / 10, T^{1 / 2} \ll H \ll T, H U \gg T^{1+\varepsilon} .
$$

Then the estimate

$$
\int_{T}^{T+H} \max _{0 \leqslant u \leqslant U}|\Delta(x+u)-\Delta(x)|^{2} \mathrm{~d} x \ll H U \log ^{c} T
$$

holds for some absolute constant $c \geqslant 0$.

According to Lemma 2.1, Conjecture 4 is true for $H=T$ with $c=5$. It is trivially implied by Conjecture 3. Nevertheless, it is very strong, since it implies Conjecture 1. Namely we have the following

Proposition 4.1. Conjecture 4 implies Conjecture 1. 
Proof. Suppose $U \ll T^{1 / 2} \ll H$. Then we have

$$
\begin{aligned}
\Delta(T) & =\frac{1}{U} \int_{T-U}^{T} \Delta(T) \mathrm{d} x \\
& =\frac{1}{U} \int_{T-U}^{T} \Delta(x) \mathrm{d} x+\frac{1}{U} \int_{T-U}^{T}(\Delta(T)-\Delta(x)) \mathrm{d} x \\
& \ll \frac{1}{U}\left|\int_{T-U}^{T} \Delta(x) \mathrm{d} x\right|+\frac{1}{U} \int_{T-U}^{T}|\Delta(T)-\Delta(x)| \mathrm{d} x \\
& \ll \frac{U+T^{3 / 4}}{U}+\frac{1}{U} \int_{T-U}^{T}|\Delta(x+T-x)-\Delta(x)| \mathrm{d} x \\
& \ll \frac{U+T^{3 / 4}}{U}+\frac{1}{U} \int_{T-U}^{T} \max _{0 \leqslant u \leqslant U}|\Delta(x+u)-\Delta(x)| \mathrm{d} x \\
& \ll \frac{U+T^{3 / 4}}{U}+\frac{1}{U} \int_{T-H}^{T} \max _{0 \leqslant u \leqslant U}|\Delta(x+u)-\Delta(x)| \mathrm{d} x,
\end{aligned}
$$

where we used the well-known formula of Voronoï [23]

$$
\int_{0}^{T} \Delta(x) \mathrm{d} x=\frac{1}{4} T+O\left(T^{3 / 4}\right) .
$$

By (4.2), the Cauchy-Schwarz inequality and Conjecture 4 with $U=$ $T^{1 / 2-\varepsilon}$ and $H=T^{1 / 2+2 \varepsilon}$ we obtain from $(4.2)$

$$
\Delta(T) \ll_{\varepsilon} T^{1 / 4+\varepsilon}+\frac{1}{U}\left(H U \log ^{3} T\right)^{1 / 2} H^{1 / 2} \ll_{\varepsilon} T^{1 / 4+\varepsilon} .
$$

\section{A partial answer to Conjecture 4}

In this section, we shall show that the argument of Heath-Brown and Tsang [5] implies a partial answer to Conjecture 4. This is

Theorem 2. Suppose $\log ^{2} T \ll U \leqslant T^{1 / 2} / 2, T^{1 / 2} \ll H \leqslant T$, then we have

$$
\begin{aligned}
& \int_{T}^{T+H} \max _{0 \leqslant u \leqslant U}|\Delta(x+u)-\Delta(x)|^{2} \mathrm{~d} x \ll H U \mathcal{L}^{5}+T \mathcal{L}^{4} \log \mathcal{L} \\
& +H^{1 / 3} T^{2 / 3} U^{2 / 3} \mathcal{L}^{10 / 3}(\log \mathcal{L})^{2 / 3}
\end{aligned}
$$

where $\mathcal{L}:=\log T$.

Proof. Write $U=2^{\lambda} b$ where $\lambda \in \mathbb{N}$ and $1<b \leqslant U / 10$ is a parameter to be determined later. Suppose $v \leqslant u \leqslant 2 T$. By the definition of $\Delta(x)$, we have

$$
\begin{aligned}
\Delta(u)-\Delta(v) & =\sum_{v<n \leqslant u} d(n)-M(u)+M(v) \\
& \geqslant-M(u)+M(v) \\
& \geqslant-(u-v)(\log u+2 \gamma) \\
& \geqslant-3(u-v) \mathcal{L}
\end{aligned}
$$


where $M(z)=z \log z+(2 \gamma-1) z$.

Suppose $x \asymp T, 0<u \leqslant U$. Then there is some integer $j$ such that $0 \leqslant j \leqslant U / b$ and $j b<u \leqslant(j+1) b \leqslant U$. From (5.2) we have $\Delta(x+j b)-\Delta(x)-3 b \mathcal{L} \leqslant \Delta(x+u)-\Delta(x) \leqslant \Delta(x+(j+1) b)-\Delta(x)+3 b \mathcal{L}$,

which implies that

$$
\begin{aligned}
\max _{0 \leqslant u \leqslant U}|\Delta(x+u)-\Delta(x)| & \leqslant \max _{1 \leqslant j \leqslant 2^{\lambda}}|\Delta(x+j b)-\Delta(x)|+3 b \mathcal{L} \\
& =\left|\Delta\left(x+j_{0} b\right)-\Delta(x)\right|+3 b \mathcal{L}
\end{aligned}
$$

for some $1 \leqslant j_{0}=j_{0}(x) \leqslant 2^{\lambda}$, say. We write $j_{0}$ in the binary system as

$$
j_{0}=2^{\lambda} \sum_{\mu \in S} 2^{-\mu}=2^{\lambda-\mu_{1}}+2^{\lambda-\mu_{2}}+\ldots+2^{\lambda-\mu_{\ell}}
$$

for a certain set

$$
S=S(x)=\left\{\mu_{1}, \mu_{2}, \ldots, \mu_{\ell}\right\}, \quad 0 \leqslant \mu_{1}<\mu_{2}<\ldots<\mu_{\ell} \leqslant \lambda
$$

of distinct non-negative integers $\mu_{j}$. We claim that

$$
\Delta\left(x+j_{0} b\right)-\Delta(x)=\sum_{\mu \in S}\left(\Delta\left(x+(\nu+1) 2^{\lambda-\mu} b\right)-\Delta\left(x+\nu 2^{\lambda-\mu} b\right)\right),
$$

where

$$
\nu=\nu_{\mu}=\nu_{\mu}(x)=\sum_{\rho \in S, \rho<\mu} 2^{\mu-\rho}<2^{\mu} .
$$

The definition of $\nu_{\mu}$ implies that

$$
\nu_{\mu_{1}}=0, \nu_{\mu_{2}}=2^{\mu_{2}-\mu-1}, \ldots, \nu_{\mu_{\ell}}=2^{\mu_{\ell}-\mu_{1}}+\ldots+2^{\mu_{\ell}-\mu_{\ell-1}} .
$$

Then the right-hand side of (5.4) becomes

$$
\begin{aligned}
& \Delta\left(x+2^{\lambda-\mu_{1}} b\right)-\Delta(x)+\Delta\left(x+\left(2^{\mu_{2}-\mu_{1}}+1\right) 2^{\lambda-\mu_{2}} b\right)-\Delta\left(x+2^{\mu_{2}-\mu_{1}} 2^{\lambda-\mu_{2}} b\right) \\
& +\Delta\left(x+\left(2^{\mu_{3}-\mu_{2}}+2^{\mu_{3}-\mu_{1}}+1\right) 2^{\lambda-\mu_{3}} b\right)-\Delta\left(x+\left(2^{\mu_{3}-\mu_{2}}+2^{\mu_{3}-\mu_{1}}\right) 2^{\lambda-\mu_{3}} b\right)+\ldots \\
& +\Delta\left(x+\left(2^{\mu_{\ell}-\mu_{1}}+\ldots+2^{\mu_{\ell}-\mu_{\ell-1}}+1\right) 2^{\lambda-\mu_{\ell}} b\right)-\Delta\left(x+\left(2^{\mu_{\ell}-\mu_{1}}+\ldots+2^{\mu_{\ell}-\mu_{\ell-1}}\right) 2^{\lambda-\mu_{\ell}} b\right) \\
& \quad=\Delta\left(x+\left(2^{\lambda-\mu_{1}}+\ldots+2^{\lambda-\mu_{\ell}}\right) b\right)-\Delta(x)=\Delta\left(x+j_{0} b\right)-\Delta(x),
\end{aligned}
$$

since all the other terms cancel out. This establishes (5.4).

By the Cauchy-Schwarz inequality we then obtain

$$
\begin{aligned}
& \left|\Delta\left(x+j_{0} b\right)-\Delta(x)\right|^{2} \\
\leqslant & |S| \sum_{\mu \in S}\left(\Delta\left(x+(\nu+1) 2^{\lambda-\mu} b\right)-\Delta\left(x+\nu 2^{\lambda-\mu} b\right)\right)^{2} .
\end{aligned}
$$


Collecting all possible $\mu$ 's and $\nu$ 's, we get

$$
\begin{aligned}
& \left|\Delta\left(x+j_{0} b\right)-\Delta(x)\right|^{2} \\
\leqslant & (\lambda+1) \sum_{\mu \leqslant \lambda} \sum_{0 \leqslant \nu<2^{\mu}}\left(\Delta\left(x+(\nu+1) 2^{\lambda-\mu} b\right)-\Delta\left(x+\nu 2^{\lambda-\mu} b\right)\right)^{2} .
\end{aligned}
$$

Note that now the double sum on the right-hand side of (5.5) is independent of $x$. From (5.3) and (5.5) we immediately see that

$$
\begin{aligned}
& \max _{0 \leqslant u \leqslant U}|\Delta(x+u)-\Delta(x)|^{2} \\
& \ll \lambda \sum_{\mu \leqslant \lambda} \sum_{0 \leqslant \nu<2^{\mu}}\left(\Delta\left(x+(\nu+1) 2^{\lambda-\mu} b\right)-\Delta\left(x+\nu 2^{\lambda-\mu} b\right)\right)^{2}+b^{2} \mathcal{L}^{2},
\end{aligned}
$$

which implies that

$$
\begin{aligned}
& \ll \lambda \sum_{\mu \leqslant \lambda} \sum_{0 \leqslant \nu<2^{\mu}} \int_{T}^{T+H}\left(\Delta\left(x+(\nu+1) 2^{\lambda-\mu} b\right)-\Delta\left(x+\nu 2^{\lambda-\mu} b\right)\right)^{2} \mathrm{~d} x+H b^{2} \mathcal{L}^{2} \\
& \ll \lambda \sum_{\mu \leqslant \lambda} \sum_{0 \leqslant \nu<2^{\mu}} \int_{T+\nu 2^{\lambda-\mu_{b}}}^{T+H+\nu 2^{\lambda-\mu} b}\left(\Delta\left(x+2^{\lambda-\mu} b\right)-\Delta(x)\right)^{2} \mathrm{~d} x+H b^{2} \mathcal{L}^{2} .
\end{aligned}
$$

We remark that the error term $T^{1+\varepsilon}$ in $(2.1)$ can be replaced by $T \mathcal{L}^{3} \log \mathcal{L}$ if we couple the argument of Lau and Tsang [15] with Jutila's proof of (2.1). Hence similarly to $(2.3)$ we obtain, for $1 \leqslant U_{1} \leqslant T_{1}^{1 / 2} / 2 \ll H_{1} \leqslant T_{1}$, that

$$
\int_{T_{1}}^{T_{1}+H_{1}}\left(\Delta\left(x+U_{1}\right)-\Delta(x)\right)^{2} \mathrm{~d} x \ll H_{1} U_{1} \log ^{3} \frac{\sqrt{T}_{1}}{U_{1}}+T_{1} \log ^{3} T_{1} \log \log T_{1} .
$$

From (5.6) and (5.7), with $T_{1}=T+\nu 2^{\lambda-\mu} b=T+O(U)$ (since $U=2^{\lambda} b$ and $\nu<2^{\mu}$ ), we infer that

$$
\begin{aligned}
& \int_{T}^{T+H} \max _{0 \leqslant u \leqslant U}|\Delta(x+u)-\Delta(x)|^{2} \mathrm{~d} x \\
& \ll \lambda \sum_{\mu \leqslant \lambda} \sum_{0 \leqslant \nu<2^{\mu}}\left(H 2^{\lambda-\mu} b \mathcal{L}^{3}+T \mathcal{L}^{3} \log \mathcal{L}\right)+H b^{2} \mathcal{L}^{2} \\
& \ll \lambda \sum_{\mu \leqslant \lambda}\left(H 2^{\lambda} b \mathcal{L}^{3}+T 2^{\mu} \mathcal{L}^{3} \log \mathcal{L}\right)+H b^{2} \mathcal{L}^{2} \\
& \ll \lambda\left(H 2^{\lambda} b \mathcal{L}^{4}+T 2^{\lambda} \mathcal{L}^{3} \log \mathcal{L}\right)+H b^{2} \mathcal{L}^{2} \\
& \ll H 2^{\lambda} b \mathcal{L}^{5}+T 2^{\lambda} \mathcal{L}^{4} \log \mathcal{L}+H b^{2} \mathcal{L}^{2} \\
& \ll H U \mathcal{L}^{5}+T U b^{-1} \mathcal{L}^{4} \log \mathcal{L}+H b^{2} \mathcal{L}^{2} .
\end{aligned}
$$

Now Theorem 2 follows from (5.8) by taking

$$
b=C \min \left(\left(T U H^{-1} \mathcal{L}^{2} \log \mathcal{L}\right)^{1 / 3}, U / 10\right) .
$$


Here $C>0$ is a suitable constant such that one has

$$
\lambda=\frac{\log U / b}{\log 2} \in \mathbb{N} .
$$

From Theorem 2 we get the following Corollary 5.1, which is well-known but is usually proved by the method of exponential sums. For a proof of Voronoi's original estimate $\Delta(x) \ll x^{1 / 3} \log x$ without the use of exponential sums, see the first author's paper [9].

Corollary 5.1. We have the estimate

$$
\Delta(x) \ll x^{1 / 3} \log ^{5 / 3} x(\log \log x)^{1 / 3} .
$$

Proof. The proof is the same as that of Theorem 2. But this time we take $U=T^{1 / 2} / 10, H=10 T^{1 / 2}$. We omit the details.

Remark 3. The whole procedure leading to Corollary 5.1 is as follows: first we prove the Jutila type result (5.7) from a more accurate form of Lemma 3.1 (see Meurman [16]), then we prove Theorem 2, and finally we prove Corollary 5.1. The procedure begins with Voronoï's formula and is very long, but the result is a only a little stronger than $x^{1 / 3+\varepsilon}$, which is obtained directly from Lemma 3.1 by taking $N=x^{1 / 3}$. So it seems the above procedure is not interesting.

It is not the case. Note that it is well-known that $\Delta(x)$ has also the representation

$$
\Delta(x)=-2 \sum_{n \leq \sqrt{x}} \psi(x / n)+O(1),
$$

where $\psi(t)=\{t\}-1 / 2$ and $\{t\}$ is the fractional part of $t$. Actually we can prove (5.7) from (5.9) without using Lemma 3.1, following the approach given in Tsang-Zhai [22]. And then we prove Theorem 2 and the corollary. This means that we can prove Corollary 5.1 directly without using Voronoï's formula (Lemma 3.1).

From Theorem 2 we also get immediately the following Corollary 5.2, which is a partial answer to Conjecture 4 .

Corollary 5.2. Suppose that

$$
\log T \leqslant U \leqslant T^{1 / 2} / 10, T^{1 / 2} \ll H \ll T .
$$

Then Conjecture 4 holds for $c=5$ if

$$
H U^{1 / 2} \gg T \mathcal{L}^{-5 / 2} \log \mathcal{L} .
$$




\section{Sign changes of $\Delta(x)$ over short intervals}

In this section, we shall give a short interval analogue of Theorem A via Theorem 2. The result is

Theorem 3. Suppose $T, U, H$ are large parameters and $C>1$ is a large constant such that

$$
T^{131 / 416+\varepsilon} \ll U \leqslant C^{-1} T^{1 / 2} \mathcal{L}^{-5}, \quad C T^{1 / 4} U \mathcal{L}^{5} \log \mathcal{L} \leqslant H \leqslant T .
$$

Then in the interval $[T, T+H]$ there are $\gg H U^{-1}$ subintervals of length $\gg U$ such that on each subinterval one has $\pm \Delta(x) \geqslant c_{ \pm} T^{1 / 4}$ for some $c_{ \pm}>0$.

Corollary 6.1. Suppose $T, H$ are large parameters and $C>1$ is a large constant such that $C T^{3 / 4} \log \mathcal{L} \leqslant H \leqslant T$. Then in the interval $[T, T+H]$ there are $\gg H T^{-1 / 2} \mathcal{L}^{5}$ subintervals of length $\gg T^{1 / 2} \mathcal{L}^{-5}$ such that on each subinterval one has $\pm \Delta(x) \geqslant c_{ \pm} T^{1 / 4}$ for some $c_{ \pm}>0$.

Proof. We consider only the case of the " + " sign, and follow the method of proof of Tsang and Zhai [22]. Since $U \gg T^{131 / 416+\varepsilon}$, the condition $H \geqslant$ $C T^{1 / 4} U \mathcal{L}^{5} \log \mathcal{L}$ implies $H \gg T^{235 / 416+\varepsilon}$. Thus by Theorem 2 of Lau and Tsang [15] we have, as $T \rightarrow \infty$,

$$
\int_{T}^{T+H}|\Delta(x)|^{2} \mathrm{~d} x=C_{2} H T^{1 / 2}(1+o(1))
$$

and

$$
\int_{T}^{T+H}|\Delta(x)|^{3} \mathrm{~d} x=C_{2} H T^{3 / 4}(1+o(1)),
$$

where $C_{2}, C_{3}$ are suitable positive constants. From (6.1), (6.2) and the Cauchy-Schwarz inequality we have

$$
\begin{aligned}
H T^{1 / 2} & \ll \int_{T}^{T+H}|\Delta(x)|^{2} \mathrm{~d} x=\int_{T}^{T+H}\left|\Delta^{3 / 2}(x) \Delta^{1 / 2}(x)\right|^{2} \mathrm{~d} x \\
& \ll\left(\int_{T}^{T+H}|\Delta(x)|^{3} \mathrm{~d} x\right)^{1 / 2}\left(\int_{T}^{T+H}|\Delta(x)| \mathrm{d} x\right)^{1 / 2} \\
& \ll H^{1 / 2} T^{3 / 8}\left(\int_{T}^{T+H}|\Delta(x)| \mathrm{d} x\right)^{1 / 2},
\end{aligned}
$$

which implies that

$$
\int_{T}^{T+H}|\Delta(x)| \mathrm{d} x \gg H T^{1 / 4}
$$


From (4.3) we get

$$
\int_{T}^{T+H} \Delta(x) \mathrm{d} x \ll H+T^{3 / 4}
$$

For any $x \asymp T$, define

$$
\Delta_{+}(x)= \begin{cases}\Delta(x) & \text { if } \Delta(x)>0 \\ 0 & \text { otherwise }\end{cases}
$$

We can therefore write

$$
\int_{T}^{T+H}|\Delta(x)| \mathrm{d} x=2 \int_{T}^{T+H} \Delta_{+}(x) \mathrm{d} x-\int_{T}^{T+H} \Delta(x) \mathrm{d} x .
$$

Then from (6.3), (6.4) and the Cauchy-Schwarz inequality we get

$$
\begin{aligned}
H T^{1 / 4} & \ll \int_{T}^{T+H}|\Delta(x)| \mathrm{d} x \\
& \ll\left(\int_{T}^{T+H} \mathrm{~d} x\right)^{1 / 2}\left(\int_{T}^{T+H}\left|\Delta_{+}(x)\right|^{2} \mathrm{~d} x\right)^{1 / 2} \\
& \ll H^{1 / 2}\left(\int_{T}^{T+H}\left|\Delta_{+}(x)\right|^{2} \mathrm{~d} x\right)^{1 / 2}
\end{aligned}
$$

which implies that

$$
\int_{T}^{T+H}\left|\Delta_{+}(x)\right|^{2} \mathrm{~d} x \gg H T^{1 / 2}
$$

Finally let us define

$$
\omega(x)=\left|\Delta_{+}(x)\right|^{2}-4 \max _{0 \leqslant u \leqslant U}|\Delta(x+u)-\Delta(x)|^{2}-\delta x^{1 / 2},
$$

where $\delta>0$ is a sufficiently small positive constant. If $\omega(x)>0$, then it follows that

$$
\Delta(x) \geq \sqrt{\delta} x^{1 / 4}
$$

and

$$
\Delta(x) \geq 2 \max _{0 \leqslant u \leqslant U}|\Delta(x+u)-\Delta(x)| .
$$

The second inequality implies that for any $0 \leqslant u \leqslant U$,

$$
\frac{1}{2} \Delta(x) \leqslant \Delta(x+u) \leqslant \frac{3}{2} \Delta(x),
$$

namely $\Delta(x+u)$ has the same sign as $\Delta(x)$. 
Under the conditions of Theorem 3, from (6.5) and Theorem 2 we see that

$$
\begin{aligned}
& \int_{T}^{T+H} \omega(x) d x \\
\gg & H T^{1 / 2}-C_{2} \delta H T^{1 / 2} \\
& -C_{1}\left(H U \mathcal{L}^{5}+T \mathcal{L}^{4} \log \mathcal{L}+H^{1 / 3} T^{2 / 3} U^{2 / 3} \mathcal{L}^{10 / 3}(\log \mathcal{L})^{2 / 3}\right) \\
\gg & H T^{1 / 2}
\end{aligned}
$$

for sufficiently small $\delta$ and some absolute constants $C_{1}$ and $C_{2}$.

Let $\mathscr{S}=\{t \in[T, T+H]: \omega(x)>0\}$. By (6.6), Hölder's inequality and (6.2) we get

$$
\begin{aligned}
H T^{1 / 2} & \ll \int_{T}^{2 T} \omega(x) \mathrm{d} x \leqslant \int_{\mathscr{S}} \omega(x) d x \leqslant \int_{\mathscr{S}} \Delta_{+}^{2}(x) \mathrm{d} x \\
& \leqslant|\mathscr{S}|^{1 / 3}\left(\int_{T}^{2 T}|\Delta(x)|^{3} d x\right)^{2 / 3} \ll|\mathscr{S}|^{1 / 3} H^{2 / 3} T^{1 / 2}
\end{aligned}
$$

which implies $|\mathscr{S}| \gg H$. This completes the proof of Theorem 3 .

\section{On a problem of Tsang}

In 2010, Tsang wrote a well-written survey paper [21] about $\Delta(x)$, in which he proposed the following

Problem. Do there exist intervals $[T, T+H], H=T^{\beta}$ with $\beta>1 / 4$ such that

$$
\int_{T}^{T+H}|\Delta(x)| \mathrm{d} x \ll H T^{1 / 4-\delta}
$$

for some small positive $\delta>0$ ?

Note that, by [9], for suitable $C>0$ the interval $[T, T+C \sqrt{T}]$ contains a point $x_{0}$ where $\Delta(x)$ changes sign, hence $\Delta\left(x_{0}\right) \ll_{\varepsilon} x^{\varepsilon}$. But then, since $\Delta(x+U)-\Delta(x) \ll_{\varepsilon} x^{\varepsilon}(U+1)$ for any $U>0$, we have

$$
\begin{aligned}
\int_{x_{0}}^{x_{0}+H}|\Delta(x)| \mathrm{d} x & =\int_{x_{0}}^{x_{0}+H}\left|\Delta(x)-\Delta\left(x_{0}\right)+\Delta\left(x_{0}\right)\right| \mathrm{d} x \\
& \ll_{\varepsilon} \int_{x_{0}}^{x_{0}+H}\left(x_{0}^{\varepsilon} H+x_{0}^{\varepsilon}\right) \mathrm{d} x \ll_{\varepsilon} H^{2} x_{0}^{\varepsilon} \leqslant H x_{0}^{1 / 4-\delta}
\end{aligned}
$$

for $H=x_{0}^{\beta}, 0<\beta<1 / 4$, provided that $\varepsilon$ and $\delta$ are chosen sufficiently small. This shows why $\beta>1 / 4$ was assumed by Tsang in connection with (7.1). 
There is another easy case of Tsang's problem. Namely Theorem 1 of Lau and Tsang [15] implies that if $1 / 2<\beta<1$, then we have

$$
\int_{T}^{T+T^{\beta}}|\Delta(x)| \mathrm{d} x=C_{\beta} H T^{1 / 4}(1+o(1)) \quad(T \rightarrow \infty)
$$

for some constant $C_{\beta}(>0)$. The formula (7.2) obviously disproves (7.1) for $1 / 2<\beta<1$. However, it remains to prove or disprove (7.1) for $1 / 4 \leqslant \beta \leqslant$ $1 / 2$, and this is a difficult problem.

In this section we shall show if $\Delta(x)$ could have enough sign changes, then (7.1) in this range would be true.

We start by taking $N=T$ in Lemma 3.1. We have

$$
\Delta(x)=F(x)+O_{\varepsilon}\left(T^{\varepsilon}\right)
$$

where

$$
F(x):=\frac{x^{1 / 4}}{\sqrt{2} \pi} \sum_{n \leqslant T} \frac{d(n)}{n^{3 / 4}} \cos (4 \pi \sqrt{n x}-\pi / 4) .
$$

Obviously $F \in C^{\infty}[T / 2,3 T]$.

Suppose $I \subseteq[T, 2 T]$ is any subinterval such that $\Delta(x)$ changes its sign in $I$. Then we can find an $x \in I$ such that $F(x)=0$ or at least $|F(x)| \ll_{\varepsilon} T^{\varepsilon}$. Further suppose that $\left\{x_{r}\right\}_{r=1}^{R}$ is a sequence of points such that $T<x_{1}<$ $x_{2}<\cdots<x_{R}<2 T$ and

$$
\begin{aligned}
& F\left(x_{j}\right) \ll_{\varepsilon} T^{\varepsilon}, j=1,2, \cdots, R, \\
& \left|x_{i}-x_{j}\right| \geqslant H_{0}, \quad 1 \leqslant i<j \leqslant R,
\end{aligned}
$$

where $1 \ll H_{0} \ll T^{1 / 2} \ll R \ll T$.

Let $2<H \leqslant H_{0} / 2$. For each $1 \leqslant j \leqslant R$, we have for $x_{j} \leqslant x \leqslant x_{j}+H$ that

$$
\begin{aligned}
F(x) & =F(x)-F\left(x_{j}\right)+F\left(x_{j}\right) \\
& =F\left(x_{j}+x-x_{j}\right)-F\left(x_{j}\right)+F\left(x_{j}\right) \\
& \ll_{\varepsilon} \max _{0 \leqslant h \leqslant H}\left|F\left(x_{j}+h\right)-F\left(x_{j}\right)\right|+T^{\varepsilon} \\
& \ll_{\varepsilon} \max _{0 \leqslant h \leqslant H}|F(x+h)-F(x)|+T^{\varepsilon} .
\end{aligned}
$$


So by (7.3), (7.6) and Lemma 2.1 we have that

$$
\begin{aligned}
& \sum_{j=1}^{R} \int_{x_{j}}^{x_{j}+H}|F(x)|^{2} \mathrm{~d} x \\
\ll_{\varepsilon} & \sum_{j=1}^{R} \int_{x_{j}}^{x_{j}+H}\left(\max _{0 \leqslant h \leqslant H}|F(x+h)-F(x)|\right)^{2} \mathrm{~d} x+R T^{2 \varepsilon} \\
\ll_{\varepsilon} & \int_{T}^{2 T}\left(\max _{0 \leqslant h \leqslant H}|F(x+h)-F(x)|\right)^{2} \mathrm{~d} x+R T^{2 \varepsilon} \\
\ll_{\varepsilon} & H T \log ^{5} T+R T^{2 \varepsilon} .
\end{aligned}
$$

Formula (7.7) implies that there is some $1 \leqslant j_{0} \leqslant R$ such that

$$
\int_{x_{j_{0}}}^{x_{j_{0}}+H}|\Delta(x)|^{2} \mathrm{~d} x \ll_{\varepsilon} H T R^{-1} \log ^{5} T+T^{2 \varepsilon},
$$

which combined with the Cauchy-Schwarz inequality yields

$$
\int_{x_{j_{0}}}^{x_{j_{0}}+H}|\Delta(x)| \mathrm{d} x \ll_{\varepsilon} H T^{1 / 2} R^{-1 / 2} \log ^{5 / 2} T+H^{1 / 2} T^{\varepsilon} .
$$

So if we can take

$$
R \gg T^{1 / 2+\delta},
$$

then Tsang's problem is completely solved.

Remark 4. Since $\Delta(x)$ has sign changes in the interval $[T, T+C \sqrt{T}]$ for some absolute constant $C$, it is seen that we can take $R \gg T^{1 / 2}$ in the above argument. However, this is still far from solving Tsang's problem.

There is another approach to Tsang's problem, which will be briefly presented now. By Lemma 2 of Heath-Brown and Tsang [5], there are long intervals where $\Delta(x)$ does not change sign. In particular, there are intervals of length $\gg \sqrt{T} \log ^{-5} T$ in $[T, 2 T]$ where $\Delta(x)$ does not change sign. The same is true of

$$
F(x)=F_{N}(x):=\sum_{n \leqslant N} d(n) n^{-3 / 4} \cos (4 \pi \sqrt{n x}-\pi / 4),
$$

where

$$
T \leqslant x \leqslant 2 T, N=T^{1 / 2+2 \varepsilon+2 \delta} .
$$

Then there exists an interval $\left[X_{0}-H, X_{0}+2 H\right]$, where $F\left(X_{0}-H\right)=0$, for $H$ satisfying $H \ll \sqrt{T} \log ^{-5} T$, where $F_{N}(x)$ does not change sign. Let $\varphi(x)(\geqslant 0)$ be a smooth function supported in $\left[X_{0}-H, X_{0}+2 H\right]$ such that $\varphi(x)=1$ in $\left[X_{0}, X_{0}+H\right]$ and $\varphi^{(r)}(x) \ll_{r} H^{-r}$. Then we have

$$
\int_{X_{0}}^{X_{0}+H}|\Delta(x)| \mathrm{d} x \leqslant \int_{X_{0}}^{X_{0}+2 H} \varphi(x)|\Delta(x)| \mathrm{d} x=\left|\int_{X_{0}}^{X_{0}+2 H} \varphi(x) \Delta(x) \mathrm{d} x\right|
$$




$$
=\frac{1}{\pi \sqrt{2}}\left|\int_{X_{0}-H}^{X_{0}+2 H} x^{1 / 4} \varphi(x) F_{N}(x) \mathrm{d} x\right|+O\left(H T^{1 / 4-\delta}\right) .
$$

We integrate sufficiently many times the last integral by parts, getting each time the same type of exponential integral, with a new factor of order $\ll$ $T^{1 / 2} H^{-1} n^{-1 / 2}$ in the $n$-th term in $F_{N}(x)$. This means that we may truncate $F_{N}(x)$ at $N=T^{1+\varepsilon} H^{-2}$, or in other words replace $F_{N}(x)$ by $F_{M}(x), M=$ $T^{1+\varepsilon} H^{-2}$. The point is that, besides the fact that there are no absolute value signs in the integral, the sum $F_{M}(x)$ is shorter than $F_{N}(x)$, which is significant. Also one should be able to use the fact that $F\left(X_{0}-H\right)=0$ to show that, for $n$ not large, the initial terms in $F_{M}(x)$ and $F_{N}\left(X_{0}-H\right)$ are small. Namely one cannot make use directly of $F_{N}(x)$, even for small $n$, and show that their contribution is $O\left(H T^{1 / 4-\delta}\right)$. But the initial terms in $F_{M}(x)-F_{N}\left(X_{0}-H\right)$ are small if $x$ is close to $X_{0}$. This ought to be taken into account to show that Tsang's conjecture holds true. If we can prove that

$$
\int_{X_{0}-H}^{X_{0}+2 H} x^{1 / 4} \varphi(x) F_{N}(x) \mathrm{d} x \ll H T^{1 / 4-\delta}
$$

with $N=M=T^{1+\varepsilon} H^{-2}$ and $T^{1 / 4} \ll H \ll \sqrt{T} \log ^{-5} T$, then we are done. 


\section{References}

[1] H. Cramér, Über zwei Sätze von Herrn G. H. Hardy, Math. Z. 15(1922), 201-210.

[2] S.W. Graham and G. Kolesnik, Van der Corput's method of exponential sums, LMS Lecture Notes series 126, Cambridge University Press, Cambridge, 1991.

[3] D.R. Heath-Brown, The mean value theorem for the Riemann zetafunction, Mathematika 25(1978), 177-184.

[4] D. R. Heath-Brown, The distribution and moments of the error term in the Dirichlet divisor problem, Acta Arith. 60(1992), 389-415.

[5] D. R. Heath-Brown and K.Tsang, Sign changes of $E(t), \Delta(x)$ and $P(x)$, J. of Number Theory 49(1994), 73-83.

[6] M. N. Huxley, Exponential sums and Lattice points III, Proc. London Math. Soc. 87(2003), 591-609.

[7] Large values of the error term in the divisor problem, Inventiones Math. 71(1983), 513-520.

[8] A. Ivić, The Riemann zeta-function. John Wiley and Sons, New York, 1985.

[9] A. Ivić, Large values of certain number-theoretic error terms, Acta Arith. 56(1990), 135-159.

[10] A. Ivić, The circle and divisor problem, Bulletin CXXIX de l'Académie Serbe des Sciences et des Arts - 2004, Classe des Sciences mathématiques et naturelles, Sciences mathématiques No. 29, pp. 7983.

[11] A. Ivić, On the divisor function and the Riemann zeta-function in short intervals, The Ramanujan Journal: Volume 19, Issue 2 (2009), 207-224.

[12] A. Ivić and P. Sargos, On the higher power moments of the error term in the divisor problem, Illinois J. of Math. 81(2007), 353-377.

[13] A. Ivić and W. Zhai, Higher moments of the error term in the divisor problem (in Russian), Matematičeskie Zametki 88(2010), 374-383 (= Math. Notes 88(2010), 338-346). 
[14] M. Jutila, On the divisor problem for short intervals, Ann. Univ. Turkuensis Ser. AI 186(1984), 23-30.

[15] Y.-K. Lau and K.-M. Tsang, On the mean square formula of the error term in the Dirichlet divisor problem, Math. Proc. Camb. Phil. Soc., Vol. 146(2009), 277-287.

[16] T. Meurman, On the mean square of the error term in a generalization of Dirichlet's divisor problem. Acta Arith. 74(1996), 351-364.

[17] E. Preissman, Sur la moyenne de la fonction zêta. Nagasaka, Kenji (ed.), Analytic number theory and related topics. Proceedings of the symposium, Tokyo, Japan, November 11-13, 1991. Singapore: World Scientific, 119-125 (1993).

[18] O. Robert and P. Sargos, Three-dimensional exponential sums with monomials, J. reine angew. Math. 591(2006), 1-20.

[19] K. C. Tong, On divisor problem III, Acta math. Sinica 6(1956), 515541.

[20] K.-M. Tsang, Higher-power moments of $\Delta(x), E(t)$ and $P(x)$, Proc. London Math. Soc.(3) 65(1992), 65-84.

[21] K.-M. Tsang, Recent progress on the Dirichlet divisor problem and the mean square of the Riemann zeta-function, Science China Mathematics, Vol. 53(2010), 2561-2572.

[22] K.-M. Tsang and W. Zhai, Sign changes of the error term in Weyl's law for the Heisenberg manifolds, Transactions of AMS, 364(2012), Number 5, 2647-2666.

[23] G.F. Voronoï, Sur une fonction transcendante et ses applications à la sommation de quelques séries, Ann. École Normale 21(3)(1904), 207268 and ibid. 21(3)(1904), 459-534.

[24] W. Zhai, On higher-power moments of $\Delta(x)$ (II), Acta Arith. 114(2004), 35-54.

A. Ivić, Katedra Matematike RGF-a Universiteta u Beogradu, DuUŠINA 7, 11000 BeOgrad, SERBIA. ivic@rgf.bg.ac.rs

W. Zhai, Department of Mathematics, China University of Mining And Technology, Beijing 100083, China. zhaiwg@hotmail.com 